2 Cropsey C, Kennedy J, Han J, et al. Cognitive dysfunction, delirium, and stroke in cardiac surgery patients. Semin Cardiothorac Vasc Anesth 2015;19:309-17.

3 Evered L, Silbert B, Knopman DS. (Nomenclature Consensus Working Group), et al. Recommendations for the nomenclature of cognitive change associated with anaesthesia and surgery-2018. Anesthesiology 2018;129:872-9.

4 Trabold B, Metterlein T. Postoperative delirium: Risk factors, prevention, and treatment. J Cardiothorac Vasc Anesth 2014;28:1352-60.

5 Kumar AK, Jayant A, Arya VK, et al. Delirium after cardiac surgery: A pilot study from a single tertiary referral center. Ann Card Anaesth 2017;20:76-82.

6 Glumac S, Kardum G, Karanovic N. Postoperative cognitive decline after cardiac surgery: A narrative review of current knowledge in 2019. Med Sci Monit 2019;25:3262-70.

Fu-Shan Xue, MD Shao-Hua Liu, MD

Hai-Jun Hou, MD Department of Anesthesiology, Beijing Friendship Hospital, Capital Medical University, Beijing, People's Republic of China

https://doi.org/10.1053/j.jvca.2020.06.067

\section{Can Intraoperative Optimization of Both Anesthetic Depth and Cerebral Oxygenation Really Result in Different Influences on Cognitive Function and Delirium in Elderly Patients Undergoing Cardiac Surgery? Response to Letter to the Editor by Xue et al.}

\section{To the Editor:}

WE WOULD like to thank Xue et al. for their letter with reference to our article about optimization of intraoperative depth of anesthesia and cerebral oxygenation.

We fully agree that recent recommendations for the nomenclature of cognitive change associated with anesthesia and surgery are important. These recommendations state that the diagnosis of postoperative delirium (POD) occurs up to 1 week after surgery or until discharge. ${ }^{2}$ The recommendations were published after our study was conducted, and assessments of POD for a longer period postoperatively may have resulted in an increased rate of POD in our patients. However, a previous study demonstrated in elderly patients that the peak of patients with POD occurred on day 3 after surgery. ${ }^{3}$ Furthermore, another observational study showed that the mean length of POD was 3.7 days (with a standard error of $0.6 \mathrm{~d}$ and a range of 1-10 d). ${ }^{4}$ The recent European Society of Anaesthesiology evidence-based guidelines define POD as an "acute and fluctuating alteration of mental state ... (which) often starts in the recovery room and occurs up to 5 days after surgery." We therefore are confident that, whereas we may have underestimated the incidence of POD by assessing POD on postoperative days 3- to- 5, we assessed POD during its peak postoperative occurrence and according to recent European Society of Anaesthesiology guidance.

DOI of original article: http://dx.doi.org/10.1053/j.jvca.2020.06.067.
We agree with Xue et al. that blood transfusion is an important independent variable for POD. We demonstrated in our Results section that the perioperative transfusion of packed red cells was similar in both study groups, with $23 \%$ in the control group and $29 \%$ in the intervention group. The hemoglobin transfusion trigger in our institution is between 70 and $80 \mathrm{~g} / \mathrm{L}$ after cardiac surgery depending on patients' comorbidities. Perioperative techniques to maintain and optimize oxygenation at the tissue levels are frequently applied in our patients per institutional protocol, including transesophageal echocardiographyand cardiac output monitoring-guided fluid therapy, inotropes, and regular monitoring and optimization of central venous oxygen saturations. With our results of similar red blood cell transfusions in both groups and a standard of care to optimize perioperative tissue oxygenation in all patients, the proposed chances of bias suggested by Xue et al. were very small. In addition, to avoid bias, the teams conducting postoperative treatments and assessments were blinded.

Finally, we agree that including our institutional pain protocol in the Methods section of the article may have been helpful. King's College Hospital's cardiac postoperative pain protocol has been described before, and it includes an opioid infusion (morphine or fentanyl) until extubation, paracetamol and oral codeine every 6 hours after extubation, and oral morphine when necessary. ${ }^{6}$ This protocol has been shown to relieve pain toward a median of mild pain scores in our patients. ${ }^{6}$ As a result of the application of this pain protocol, poor postoperative pain control was avoided in the majority of patients and thus pain- associated POD or postoperative cognitive dysfunction (POCD).

We disagree with Xue et al. that we did not present a list of postoperative complications. We listed relevant postoperative complications and lengths of hospital and critical care stays in Table 3, including postoperative infections and postoperative acute kidney injury. These were similar in both the treatment and control groups, and therefore postoperative complications were unlikely to have caused an increased incidence of delirium in 1 of the groups.

We also disagree with the authors that neurocognitive recovery should have occurred within 30 days. Mild POCD can occur until 12 months and major neurocognitive disorders beyond 12 months. $^{2}$ Therefore, we considered a primary outcome of POCD at 6 weeks as appropriate.

We again would like to thank Xue et al. for their useful comments.

D.W. Green has received funding for research (including loan equipment), honoraria for lecturing at meetings, and reimbursement of traveling expenses from Medtronic, Minneapolis, MN.

\section{References}

1 Kunst G, Gauge N, Salaunkey K, et al. Intraoperative optimization of both depth of anesthesia and cerebral oxygenation in elderly patients undergoing coronary artery bypass graft surgery-a randomized controlled pilot trial. J Cardiothorac Vasc Anesth 2020;34:1172-81. 
2 Evered L, Silbert B, Knopman DS, et al. Recommendations for the nomenclature of cognitive change associated with anaesthesia and surgery-2018. Br J Anaesth 2018;121:1005-12.

3 Olin K, Eriksdotter-Jonhagen M, Jansson A, et al. Postoperative delirium in elderly patients after major abdominal surgery. Br J Surg 2005;92:1559-64.

4 Sharma PT, Sieber FE, Zakriya KJ, et al. Recovery room delirium predicts postoperative delirium after hip-fracture repair. Anesth Analg 2005;101: 1215-20.

5 Aldecoa C, Bettelli G, Bilotta F, et al. European Society of Anaesthesiology evidence-based and consensus-based guideline on postoperative delirium. Eur J Anaesthesiol 2017;34:192-214

6 Fleming IO, Garratt C, Guha R, et al. Aggregation of marginal gains in cardiac surgery: Feasibility of a perioperative care bundle for enhanced recovery in cardiac surgical patients. J Cardiothorac Vasc Anesth 2016;30: $665-70$.

\author{
Gudrun Kunst, MD, PhD, EDAIC, FRCA, FFICM**** \\ David W. Green, MBBS, MBA, FRCA* \\ Clive Ballard, MRCPsych, MMedSci, MD, FMedSci ${ }^{\dagger}$ \\ "Department of Anaesthetics, King's College Hospital, London, \\ United Kingdom \\ ** School of Cardiovascular Medicine \& Sciences, King's College London \\ British Heart Foundation Centre of Excellence \\ ${ }^{\dagger}$ University of Exeter Medical School, Exeter, United Kingdom
}

https://doi.org/10.1053/j.jvca.2020.06.069

\section{Plasma Volume and Cardiac Surgery: Is TEG R-Time a Better Indicator Than the INR?}

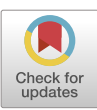

\section{To the Editor:}

We are intrigued to read Smith et al.'s robust retrospective study of plasma transfusion volumes in patients undergoing cardiac surgery. ${ }^{1}$ Plasma transfusion at this experienced blood management center was guided by algorithm and indicated by clinician consensus and deviations in plasma partial thromboplastin time $>57$ seconds and International Normalized Ratio (INR) $>1$.6. Key conclusions included association of increasing plasma volume transfusion with inferior clinical outcomes and association of higher pretransfusion INR, with increased risk for perioperative red blood cell transfusion. As the authors note, there were limitations of the INR in this clinical scenario. In this report, pre- and postoperative INR values were 1.7 (falling within a traditional range for consideration for plasma transfusion) and 1.3, respectively, with most of the cohort falling between 1.5 and 1.9 .

The limited sample size that had simultaneous Thrombelas$\operatorname{tograph}^{\mathrm{R}}$ (TEG) R-time values $(20.8 \%)$ is illuminating as both pre- $(7.3 \mathrm{~min})$ and postoperative $(6.8 \mathrm{~min})$ measures were within the normal range for kaolin- activated analysis (activator not stated in manuscript). Although the authors acknowledge the inadequate power of the TEG data subset, neither the discrete R-time nor changes in R-time were associated with the described outcomes.

Both the trigger for plasma transfusion (elevated INR) and plasma transfusion itself were associated with poorer outcomes

DOI of original article: http://dx.doi.org/10.1053/j.jvca.2019.12.049. and increased red blood cell transfusion. Because the TEG Rtime was not a marker of poor outcome, conceptually, one could presume that if the R-time was used as a guide to plasma transfusion, the normal values detected would likely have decreased indication for plasma transfusion and thus the measured outcomes.

Successful implementation of viscoelastic tests such as the TEG have been described well in cardiac surgery. ${ }^{2,3}$ The secondary outcome from this study with normal R-time in the presence of a somewhat elevated INR suggested that the Rtime may be a safer indicator of the integrity of fibrin formation, with a subsequently decreased threshold for plasma transfusion and potential adverse effects.

\section{Conflict of Interest}

All authors contributed to the manuscript and none has relevant conflicts of interest to declare. No funding was used apart from department resources.

\section{References}

1 Smith MM, Kor DJ, Frank RD, et al. Intraoperative plasma transfusion volumes and outcomes in cardiac surgery. J Cardiothorac Vasc Anesth 2020;34:1446-56.

2 Raphael J, Mazer CD, Subramani S, et al. Society of Cardiovascular Anesthesiologists clinical practice improvement advisory for management of perioperative bleeding and hemostasis in cardiac surgery patients. J Cardiothorac Vasc Anesth 2019:33;2887-99.

3 Redfern RE, Fleming K, March RL, et al. Thromboelastography-directed transfusion in cardiac surgery: Impact on postoperative outcomes. Ann Thorac Surg 2019;107:1313-8.

Travis H Markham, MD Evan G Pivalizza, MBChB, FFASA Department of Anesthesiology, UTHealth McGovern Medical School, Houston, TX

https://doi.org/10.1053/j.jvca.2020.06.080

\section{TEG R-Time Guided Plasma Transfusion: R We There Yet?}

\section{To the Editor:}

We appreciate the insightful comments by Drs. Markham and Pivalizza. ${ }^{1}$ We agree that viscoelastic testing is welldescribed and commonly used in the management of perioperative cardiac surgical bleeding. Implementation of viscoelastic-based transfusion protocols has been associated with reduced perioperative transfusions and associated complications. The same is also true for transfusion protocols based on standard tests of coagulation; hence, any standardized transfusion protocol is likely better than no protocol at all. As described in our manuscript, elevations in International Normalized Ratio (INR) and also increasing plasma transfusion

DOI of original article: http://dx.doi.org/10.1053/j.jvca.2020.06.080. 